1601d HAZARD DETERMINANTS OF CARBON NANOTUBES (CNTS) DRIVING MOLECULAR INITIATING EVENTS (MIES) IN ADVERSE OUTCOME PATHWAYS (AOPS) OF AIRWAYS DISEASES

${ }^{1} E$ Bergamaschi* ${ }^{2}{ }^{2}$ Aldieri, ${ }^{2} E$ Gazzano, ${ }^{2} E$ Polimeni, ${ }^{2} \mathrm{C}$ Riganti, ${ }^{3} \mathrm{O}$ Bussolati, ${ }^{3} \mathrm{M}$ Allegri, ${ }^{3}$ MG Bianchi, 'F Donato, ${ }^{4} \mathrm{~A}$ Marucco, ${ }^{4}$ I Fenoglio. ${ }^{\prime}$ Department of Public Health Sciences and Paediatrics, University of Turin, Turin, Italy; ${ }^{2}$ Department of Oncology, University of Turin, Turin, Italy; ${ }^{3}$ Department of Medicine and Surgery, University of Parma, Parma, Italy; ${ }^{4}$ Department of Chemistry, University of Turin, Turin, Italy

\subsection{6/oemed-2018-ICOHabstracts.801}

Introduction Because of their unique physico-chemical properties, CNTs have attracted a great deal of research interest and have many promising industrial applications. However, this also increases the exposure potential for workers, raising the need to understand their hazard for an effective occupational health and safety management. CNTs can induce lung inflammation, granuloma formation, fibrosis and cancer in rodents; in particular, MWCNTs are known to induce in vitro markers of remodelling and fibrosis. CNTs greatly vary in length, thickness, rigidity, aspect ratio, surface defects and reactivity, with a remarkable contribution of synthesis methods and posttreatments. Thus, CNTs are not a single substance, but a heterogeneous family of materials that elicit different biological responses and, thus, are associated with different hazard levels not simply ascribable to the fibre paradigm.

Methods Cell models representative of the airway barrier were challenged with MWCNT preparations endowed with different physico-chemical properties, evaluating endpoints such as viability, expression of pro-inflammatory markers, nitric oxide production, epithelial barrier competence, clonogenic activity, genotoxicity. Epithelial-mesenchymal transition (EMT) was also assessed as an early event leading to fibrosis and, possibly, involved in neoplastic transformation.

Results Only long MWCNTs promoted EMT and caused frustrated phagocytosis. On the other hand, MWCNT agglomeration led to contact-mediated focal epithelial damage and impaired barrier functionality in vitro. Functionalization with carboxyl or amino groups modified the quantity and type of proteins adsorbed and, hence, the interaction with cells.

Discussion These findings may contribute to safe-by design manufacturing of MWCNT. Importantly, all the endpoints evaluated represent MIEs than can be combined to construct putative AOPs, associated with disease onset and progression. It is therefore concluded that the knowledge of the physicochemical properties associated to the MIEs of different adverse outcomes is a pre-requisite for the toxicological profile of a MWCNT preparation.

\section{1e A NEW RISK GROUPING CONCEPT FOR HIGH ASPECT RATIO MATERIALS - ADDING FIBRE RIGIDITY TO THE PICTURE}

D Kehren*, D Broßell, A Meyer-Plath, S Plitzko. German Federal Institute for Occupational Safety and Health, Berlin, Germany

\subsection{6/oemed-2018-ICOHabstracts.802}

Introduction Identification and management of risks related to both established and innovative materials are central aims of occupational safety and health. Therefore, we propose a new grouping scheme to evaluate the risk of High Aspect Ratio Materials (HARM) according to hazard and exposure aspects.
Methods Our approach to measure the fibre rigidity (discussion) is based on frequency measurements at resonance conditions of single vibrating fibres by means of radio-frequency engineering and scanning electron microscopy; using Euler-Bernoulli's beam theory to determine the young modulus.

Results The new scheme considers both intrinsic material and handling process-related properties such as bio-durability, toxicity, respirability, HARM morphology/dimensions as well as grade of agglomeration and dust release propensity during/ after processing. It is based on the results of extensive research regarding those properties and their scalability for risk assessment, most notably the dustiness. Its utilisation requires data for the mentioned intrinsic and process related properties. Especially with respect to aspects of dustiness, this requires data on HARM release propensities for different handling conditions. However this talk will focus in the fibre rigidity as new parameter.

Discussion We propose to include the aspect of rigidity, more precisely the fibre flexural rigidity, as an extension to the fibre-toxicological paradigm as a new parameter for HARM toxicity assessment. Critical fibre rigidity is most probably the key to frustrated phagocytosis or HARM translocation and distinguishes HARM toxicology from that of granular biodurable particle materials. The potential toxicity of HARMs is widely known and was also shown for CNTs/CNF in many recent studies. We believe that the toxicity should not be evaluated solely by the fibre dimensions in context with systematic animal testing, but propose to combine a fibre's composition and diameter into the property flexural rigidity. For bio-durable HARM, rigidity has the potential to become an overarching, material independent assessment parameter.

\section{$1601 f$ A NEW RISK GROUPING CONCEPT FOR HIGH ASPECT RATIO MATERIALS - THE SHAKER DUSTINESS TEST}

D Broßell ${ }^{*}$, D Kehren, E Heunisch, V Bachmann, D Wenzlaff, A Meyer-Plath, S Plitzko. Federal Institute for Occupational Safety and Health (BAA), Berlin, Germany

\subsection{6/oemed-2018-ICOHabstracts.803}

Introduction High aspect ratio materials (HARM) like carbon nanotubes (CNT) exhibit material properties that enable innovative applications but also raised concerns about potentially harmful effects to humans due to their asbestos-like morphology. Control banding by grouping of HARM by hazard- and exposure-related properties is a promising approach to risk assessment and risk mitigation for a large family of materials. Limit values enabling a differentiation of HARM with low, moderate or high dustiness as well as information about the dust morphology are necessary for control banding.

Methods We have developed a dustiness test for powdery HARM, in particular for CNTs. In the Shaker method, a laminar low volume air flow passes through a vertically vibrating powder column resulting in powder fluidization. Vibration is required to overcome adhesive forces between powder grains that would otherwise hinder fluidization. The Shaker method combines aerosol monitoring over the dust generation process to determine the emission intensities with simultaneous dust sampling. Subsequent sample analysis by means of scanning electron microscopy (SEM) obtains information about dust morphology.

Results and discussion Using a standard operation procedure, we performed dustiness tests on 20 CNTs. The results lead to material rankings based on the emission intensity and the 